\section{Cerebrovascular} Diseases
Cerebrovasc Dis 1998;8:73-77

\title{
Secondary Prevention for Stroke after CAPRIE and ESPS-2
}

\section{Stephen M. Davis ${ }^{\mathrm{a}}$ \\ Geoffrey A. Donnan ${ }^{\mathrm{b}}$ \\ Departments of Neurology, \\ a Royal Melbourne Hospital, and \\ ${ }^{\mathrm{b}}$ Austin and Repatriation Medical Centre, \\ Melbourne, Australia}

In recent years, much of the momentum in stroke prevention has been generated by the substantial risk reduction than can be achieved by carotid endarterectomy for severe carotid disease [1] and warfarin for non-valvular atrial fibrillation [2]. These landmark trial results have perhaps overshadowed the importance of antiplatelet therapy as a mainstay of stroke prevention, since the value of aspirin was first established by pivotal studies 2 decades ago $[3,4]$. The relative risk reduction of $25 \%$ for the composite outcomes of stroke, death or myocardial infarction, achieved by aspirin as secondary prevention therapy [5], appears to be fairly modest compared with relative risk reductions of $68 \%$ for stroke using warfarin for non-valvular atrial fibrillation [2], or a similar $65 \%$ reduction in ipsilateral stroke with endarterectomy for patients with severe, symptomatic carotid stenosis [1]. However, the recent publication of the CAPRIE [6] and ESPS-2 [7] antiplatelet trial results, together with other evidence of the potential efficacy of combination antiplatelet therapies [8], has raised the prospect of significantly more effective stroke prevention than can be achieved by aspirin alone.

Previously, much of the focus in antiplatelet therapies centred around the issue of the optimal dose of aspirin, with strong advocates for those in 'low-dose' and 'higherdose' regimens [9-13]. The clinical efficacy of the lower doses was supported by the experimental evidence of effective inhibition of thromboxane $\mathrm{A}_{2}$, while allowing continued prostacyclin production [14]. A series of investigations evaluating the optimal dose of aspirin have not entirely resolved this issue, but indicated a high likelihood that there is no major difference in the protective effect of doses ranging between 30 and 1,200 mg/day [9-11].
Early trials of alternative antiplatelet strategies such as sulfinpyrazone [4] and dipyridamole $[15,16]$ indicated no significant additional benefits over aspirin. Unlike aspirin, the antiplatelet drug ticlopidine does not inhibit platelet cyclo-oxygenase, but suppresses the platelet aggregation induced by adenosine diphosphate (ADP)-fibrinogen binding, collagen, adrenaline and platelet-activating factor [17]. Ticlopidine was shown to have a significant protective effect in patients with recent thromboembolic stroke [17]. In a head-to-head comparison with 'highdose' aspirin (1,300 mg daily), in patients with transient ischaemic attack or mild stroke over 3 years, ticlopidine proved superior, with a $12 \%$ greater relative risk reduction in the primary endpoints of death or non-fatal stroke [18]. Contrasting with the mild statistical benefit of ticlopidine over aspirin, there was however an increased risk of serious adverse events, notably the $1 \%$ rate of severe, although reversible neutropenia [17]. Given the modest reduction of absolute risk with ticlopidine $(2 \%$ over 3 years), cost considerations, and the need for early blood monitoring because of the risk of neutropenia, the drug has generally been used in practise as second-line therapy in patients who are either intolerant of aspirin or who constitute aspirin failures. Furthermore, the Antiplatelet Trialists' meta-analysis found no conclusive evidence that any other antiplatelet regimen, including ticlopidine, was significantly more effective than aspirin [5]. For these reasons, aspirin has remained the first-line antiplatelet strategy for most clinicians.

The ticlopidine trials $[17,18]$ were the springboard for the CAPRIE trial [6]. Clopidogrel is chemically closely related to ticlopidine, with more potent antiplatelet effects via blockage of ADP-induced platelet aggregation,

\section{KARGER}

Fax +4161306 1234

E-Mail karger@karger.ch

www.karger.com

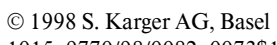


yet substantially fewer side-effects. The CAPRIE Trial was the largest clinical trial of a secondary prevention strategy to prevent serious vascular endpoints in a highrisk population. In a randomized, blinded, international trial design, the relative efficacy and safety of clopidogrel $75 \mathrm{mg}$ daily (producing ADP-induced platelet inhibition equivalent to ticlopidine $250 \mathrm{mg}$ twice daily) were compared with aspirin $325 \mathrm{mg}$ daily, in nearly 20,000 subjects comprising three groups of patients at significant risk of vascular events: those with recent ischaemic stroke; recent myocardial infarction; or symptomatic peripheral arterial disease. The primary outcome measure was a composite endpoint of ischaemic stroke, myocardial infarction or vascular death. This study showed no significant differences in adverse effects between the two antiplatelet regimens, specifically without any increased risk of neutropenia in the clopidogrel group. The results of the outcome cluster showed a significant relative risk reduction of $8.7 \%$ in favour of clopidogrel. The CAPRIE trial did not have a placebo arm. If one assumed approximately a one quarter $(25 \%)$ relative risk reduction in the aspirintreated patients based on the Antiplatelet Trialists Collaboration, the use of clopidogrel $75 \mathrm{mg}$ daily increased this to approximately one third (33\%), without increased serious adverse effects.

There were significant differences in the relative risk reductions for each of the three entry cohorts (peripheral arterial disease, stroke, myocardial infarction) in the CAPRIE Trial. The most striking effect appeared to be in patients with peripheral arterial disease, with a lack of observed benefit for clopidogrel over aspirin in the myocardial infarction subgroup and a non-significant benefit in the stroke cohort. However, the trial was powered to evaluate the overall benefits in the three subgroups. Moreover, an additional analysis of those patients in the ischaemic stroke and peripheral arterial subgroups with a previous history of myocardial infarction demonstrated clear benefits for clopidogrel over aspirin. Hence, the conclusions of this study appear to be robust and consistent with the previous ticlopidine results [18].

While the CAPRIE results showed a statistically significant benefit over aspirin, the absolute risk reduction was only $0.5 \%$, so that 200 patients per year would need to be treated with clopidogrel rather than aspirin to save one endpoint. It seems probable that clopidogrel will replace ticlopidine, because of its lack of serious adverse effects and comparable efficacy. However, it seems less likely to replace aspirin as first-line therapy in clinical practise, given its only modest biological superiority and presumed higher cost.
Dipyridamole reduces platelet aggregation by raising the anti-aggregating effects of cyclic adenosine monophosphate and cyclic guanosine monophosphate [7]. ESPS-1 (the first European Stroke Prevention Study) was published in 1987 and showed that the combination of dipyridamole $75 \mathrm{mg}$ three times daily and aspirin $330 \mathrm{mg}$ three times daily produced a $38 \%$ relative risk reduction in stroke outcomes, compared with a placebo-control group [19]. However, despite the seemingly impressive reduction in relative risk, this study could not confirm an independent effect of dipyridamole. Moreover, earlier studies of the drug had been negative, although they were probably under-powered $[5,15,16]$.

ESPS-2 was a large study of over 6,000 patients with prior stroke or transient ischaemic attack, randomised in a factorial design to treatment with very-low-dose aspirin ( $25 \mathrm{mg}$ twice daily), modified release dipyridamole (200 mg twice daily), the two agents in a combined formulation, or placebo [7]. Primary endpoints were stroke, death or stroke and death together. This study demonstrated a highly significant and independent protective effect for both aspirin and dipyridamole. In comparison with placebo, relative stroke risk was reduced by $18 \%$ with aspirin alone, $16 \%$ with dipyridamole alone and a seemingly additive $37 \%$ with the combination therapy. Factorial analysis demonstrated an independent effect of each antiplatelet agent, without evidence of statistical interaction, supporting an additive effect achieved by blockade of these different antiplatelet pathways.

There have been a number of criticisms of both the design and conduct of this large study. Some investigators have criticised the choice of the very low dose of aspirin (50 $\mathrm{mg}$ daily) used in the trial, believing that a higher dose may have produced greater risk reduction. Approximately $25 \%$ of patients were withdrawn from the trial, particularly in the dipyridamole and combination groups, chiefly due to adverse events, including headache and gastrointestinal symptoms. Moreover, compliance was confirmed in only $84 \%$ of the patients assigned to aspirin, yet $97 \%$ of those taking dipyridamole [7]. There have also been concerns about the conduct of the trial, particularly randomisation of patients to a placebo arm, during a time when most would have considered that the benefits of aspirin had already been fairly clearly established [20]. However, the results of ESPS-1 and ESPS-2 together are thought provoking and consistent, suggesting a superior rate of protection of stroke or death with the dipyridamole/ asprin combination, than aspirin alone.

For stroke prevention, other combination antiplatelet strategies appear potentially attractive. Interesting data 
exist on the combination of ticlopidine and aspirin. In laboratory studies, this combination was shown to have synergistic effects [21,22]. The combination of ticlopidine and aspirin has been evaluated in a number of stenting studies. In one study evaluating cardiac events, haemorrhagic or vascular complications after coronary artery stenting, the combination of ticlopidine and aspirin proved superior to conventional anticoagulation plus aspirin [8]. A combination trial of clopidogrel and aspirin would be a logical extension of this strategy.

The results of CAPRIE and ESPS-2 have, therefore, added significantly to the therapeutic armamentarium of antiplatelet strategies in stroke prevention. Should either clopidogrel or the dipyridamole/aspirin combination now be recommended as initial therapy for antiplatelet stroke prevention? It can be reasonably concluded that clopidogrel provides a small but significant benefit over aspirin, that dipyridamole alone seems to have an independent stroke prevention action, and that there appears to be an additive effect of the combination of aspirin and dipyridamole. However, we suspect that aspirin will continue to be used by most clinicians as their first-line stroke prevention strategy because of the small additional benefits of these other antiplatelet therapies in terms of absolute risk reduction, cost factors, side-effects in the case of dipyridamole and some reservations about the ESPS- 2 trial. In some ways, this issue is analogous to the question of the benefit of carotid endarterectomy for asymptomatic stenosis, where one trial suggested a statistically significant relative risk reduction, but a rather minimal biological benefit [23].

In patients who fail initial aspirin therapy, it certainly seems appropriate to resort to either clopidogrel, which would probably be our preference, or use combined aspirin and dipyridamole. By inference, clopidogrel and combined dipyridamole/aspirin therapy seem to have similar protective effects. However, there are obvious attractions in the use of monotherapy and a once-daily dose regimen.

Further antiplatelet trials seem likely. The platelet glycoprotein IIb/IIIa complex is the final common pathway of platelet aggregation. Oral platelet GpIIb/IIIa antagonists have great potential. They prevent the binding of fibrinogen to platelets, inhibiting platelet aggregation irrespective of the initiating metabolic pathway [24, 25]. We envisage a bright future for antiplatelet strategies in stroke prevention using new combination antiplatelet strategies or novel new approaches, such as these. Should these approaches prove to be as effective as suggested, there is a real prospect of changing clinical practise and replacing aspirin as first-line therapy.

Stephen M. Davis, MD, FRACP

Department of Neurology, Royal Melbourne Hospital

Melbourne, Vic. 3050 (Australia)

Tel. 6139342 8448, Fax 61393428427
Mark L. Dyken

Department of Neurology,

Indiana University School of Medicine,

Indianapolis, Ind., USA

On the surface the CAPRIE study [6] of clopidogrel and the ESPS-2 study [7] for dipyridamole (DP) plus aspirin (ASA) appear to have established a beneficial effect of two additional platelet antiaggregants over ASA for secondary prevention after transient ischemic attacks and stroke. But, to place these studies in perspective for clinical practice, it is necessary to review the experimental design and the actual results of each and how they fit in with other studies of platelet antiaggregating agents. After this review, I will respond to the questions posed by the editors.

The CAPRIE study is a well-designed, prospective, double-blind, controlled study in which $75 \mathrm{mg}$ /day clopi-

Secondary Prevention for Stroke after CAPRIE and ESPS-2 dogrel was compared to $325 \mathrm{mg} /$ day ASA in 19,185 patients who presented with either recent ischemic stroke, myocardial infarction or peripheral arterial disease. The primary endpoints of ischemic stroke, myocardial infarction or vascular death were decreased $8.7 \%$ /year by clopidogrel, but this was only from 5.83 to $5.32 \%$ a year. The $\mathrm{p}$ value was a modest 0.043 . If the same differences were present with 1,500 patients as planned in the original design, this would not have been significant. A test of heterogeneity of the treatment effects $(p=0.042)$ suggested that the true benefit was not identical across the three clinical subgroups. The effect was overwhelmingly in the peripheral arterial disease group. Regardless, clopidogrel 
was at least as effective as $325 \mathrm{mg}$ of ASA a day. Except for severe rash $(6.02 \%)$ and diarrhea (4.46\%), clopidogrel was equivalent to or had fewer complications than ASA. Gastrointestinal hemorrhage was minimally but statistically significantly less frequent with ASA $(1.99 \%$ vs. $2.66 \%)$.

Although ESPS-2 as reported appears to be a good study, it has been challenged for violation of ethics and fraud which may or may not affect the conclusions made by the investigators [20]. The ethics of using placebo controls was questioned because ASA has been established to be superior to placebo by many studies. The same investigators reported in ESPS-1 [19] a significant decrease in stroke for ASA and DP compared to placebo. Also, a total of 438 fictious patients from one center were excluded from analysis. Assuming all other patients entered met the protocol requirements, despite the ethical issues, one must conclude that the results reported are valid within the limits of the experimental design. Yet, the conclusions must be placed in perspective to other studies of ASA and other studies of ASA plus DP.

DP combined with ASA was compared to ASA alone for the secondary prevention of stroke in three studies, two French studies and an American-Canadian [15, 16, 26]. In none was an additional benefit shown by adding DP to ASA. In the ESPS-2, 6,602 patients were randomly assigned to take either, DP (200 mg twice a day), ASA (25 mg twice a day), a combination of ASA and DP, and placebo. At these doses, DP plus ASA was significantly superior to each of the other groups. Unfortunately, the dosages of ASA and DP were different from those used in any other study of stroke or those used by the same investigators in ESPS-1. Although doses as low as $75 \mathrm{mg}$ /day of ASA probably are effective in the secondary prevention of stroke and death, no study has shown that doses of $325 \mathrm{mg}$ a day or lower are as effective as $975 \mathrm{mg}$ /day or more [12]. In ESPS-1 a combination of ASA and DP resulted in a significant reduction in stroke and death as compared to those receiving placebo. Yet, the doses of ASA and DP were changed drastically in the second study. DP was increased almost twofold from 225 to $400 \mathrm{mg}$ a day, and ASA decreased almost 20 times from 975 to only $50 \mathrm{mg}$ a day. This may have biased the study in favor of DP, and it is quite possible that the wrong dose of ASA was used. The reduction in stroke and death $(13 \%)$ and stroke alone (18\%) for those receiving only $50 \mathrm{mg}$ a day of ASA was quite modest as compared to the high-dose studies. In fact, in ESPS-2 for those receiving ASA and DP, the reduction of stroke and death was only $24 \%$ over placebo as compared to $33 \%$ in ESPS- 1 where much higher doses of ASA were used. For stroke alone the decrease was 37 compared to $38 \%$. These differences are in the same range as those of the other high-dose studies of ASA that showed an effect $(26-42 \%)$. Thus, this study does not establish that DP in any combination is superior to ASA at any dose. It does, for the first time, suggest that DP might potentiate the effect of very-low-dose ASA to that of higher doses. The three studies that showed no additional effect by adding DP used $900-1,300 \mathrm{mg}$ of aspirin a day.

With this as background, I will respond to the charge of the editors. The results of the CAPRIE and ESPS-2 studies do not change my first choice for the secondary prevention of stroke. For patients without contraindication to ASA, this is $900-1,300 \mathrm{mg}$ of buffered or enteric coated ASA a day in divided doses. If this is not tolerated, the dose is decreased to the highest tolerable dose until $75 \mathrm{mg}$ a day before switching to another agent. Although ticlopidine is superior to ASA, its association with neutropenia, diarrhea, and its higher cost have resulted in ASA as first choice. For patients who have contraindications to ASA, such as gastrointestinal ulcer or bleeding, my primary choice would be clopidogrel because of its minimal complications. If the patient is at very high risk for stroke with many risk factors, ticlopidine would be the first choice. I would not use DP because of the dose issue, among other things, and the lack of effect in all studies except ESPS-2.

Finally, for the future it is tempting to recommend testing a combination of clopidogrel and ASA or ticlopidine and ASA because of their different effects on platelets. Despite the bias for low-dose ASA, a comparison of ASA in doses of $325 \mathrm{mg}$ a day or lower to $975 \mathrm{mg}$ a day or higher is needed with numbers large enough to avoid type II error. It might be possible that in the future, when new agents or combination of agents are being tested against ASA controls, that the ASA arm be divided into high-dose and low-dose components. The low-dose, high-dose ASA controversy cannot be solved by debate, but only by proper studies.

M.L. Dyken, MD

Department of Neurology

Indiana University School of Medicine

BA 402, 1001 West 10th Street

Indianapolis, IN 46202-2879 (USA)

Tel. (317) 274 0218, Fax (317) 6307910 $\overline{76} \quad \overline{\text { Cerebrovasc Dis 1998;8:73-77 }}$
Opinion 2 


\section{References}

1 North American Symptomatic Carotid Endarterectomy Trial Collaborators: Beneficial effect of carotid endarterectomy in symptomatic patients with high grade carotid stenosis. N Engl J Med 1991;325:445-453.

2 Atrial Fibrillation Investigators: Risk factors for stroke and efficacy of antithrombotic therapy in atrial fibrillation. Analysis of pooled data from five randomized controlled trials. Arch Intern Med 1994;154:1449-1457.

3 Fields WS, Lemak NA, Frankowski RF, Hardy RJ: Controlled trial of aspirin in cerebral ischemia. Stroke 1977;8:301-314.

4 Canadian Co-Operative Study Group: A randomized trial of aspirin and sulfinpyrazone in threatened stroke. N Engl J Med 1978;299:5359.

5 Antiplatelet Triallists Collaboration: Collaborative overview of randomised trials of antiplatelet therapy -1 . Prevention of death, myocardial infarction, and stroke by prolonged antiplatelet therapy in various categories of patients. BMJ 1994;308:81-106.

6 CAPRIE Steering Committee: A randomised, blinded, trial of clopidogrel versus aspirin in patients at risk of ischaemic events (CAPRIE). Lancet 1996;348:1329-1339.

7 Diener H, Cunha L, Forbes C, Sivenius J, Smets P, Lowenthal A: European Stroke Prevention Study 2. Dipyridamole and acetylsalicylic acid in the secondary prevention of stroke. J Neurol Sci 1996;143:1-13,

8 Schomig A, Neumann FJ, Kastrati A, Schuhlen H, Blasini R, Hadamitzky M, Walter H, Zitzmann-Roth EM, Richardt G, Alt E, Schmitt C, Ulm K: A randomized comparison of antiplatelet and anticoagulant therapy after the placement of coronary-artery stents. N Engl J Med 1996;334:1084-1089.
9 UK-TIA Study Group: United Kingdom transient ischemic attack (UK-TIA) aspirin trial; Interim results. Br Med J 1988;296:316-320.

10 Dutch TIA Trial Study Group: A comparison of two doses of aspirin ( $30 \mathrm{mg}$ vs $283 \mathrm{mg}$ a day) in patients after a transient ischemic attack or minor ischemic stroke. N Engl J Med 1991; 325:1261-1266.

11 The SALT Collaborative Group: Swedish Aspirin Low-Dose Trial (SALT) of $75 \mathrm{mg}$ aspirin as secondary prophylaxis after cerebrovascular ischaemic events. Lanced 1991;338:13451349.

12 Dyken ML, Barnett HJM, Easton JD, Fields WS, Fuster V, Hachinski V, Norris JW, Sherman DG: Low-dose aspirin and stroke. 'It ain't necessarily so' (editorial). Stroke 1992;23: 1395-1399.

13 Easton JD: Antiplatelet therapy in the prevention of stroke. Drugs 1992;42(suppl 5):3950 .

14 Patrono C: Aspirin as an antiplatelet drug. N Engl J Med 1994;330:1287-1293.

15 Bousser MG, Eschwege E, Haguenau M, Lefaucconnier JM, Thibult N, Touboul D, Touboul PJ: 'AICLA' controlled trial of aspirin and dipyridamole in the secondary prevention of atherothrombotic cerebral ischemia. Stroke 1983;14:5-14.

16 American Canadian Cooperative Study Group: Persantine Aspirin Trial in cerebral ischemia. II. Endopoint results. Stroke 1985;16:406415.

17 Gent M, Blakely JA, Easton JD, Ellis DJ, Hachinski VC, Harbison JW, Panak E, Roberts RS, Sicurella J, Turpie AGG: The Canadian American Ticlopidine Study (CATS) in thromboembolic stroke. Lancet 1989;i:1215-1220.
18 Hass WK, Easton JD, Adams HP Jr, PrysePhillips W, Molony BA, Anderson S, Kamm B: A randomized trial comparing ticlopidine hydrochloride with aspirin for the prevention of stroke in high-risk patients. N Engl J Med 1989;321:501-507.

19 European Stroke Prevention Study Group: The European Stroke Prevention Study (ESPS). Principal endpoints. Lancet 1987;ii:13511354.

20 Enserink M: Clinical trials: Fraud and ethics charges hit stroke drug trial. Science 1996;274: 2004-2005.

21 Uchiyama S, Sone R, Nagayama T, Shibagaki Y, Kobayashi I, Maruyama S, Kusakabe K: Combination therapy with low-dose aspirin and ticlopidine in cerebral ischemia. Stroke 1989;20:1643-1647.

22 DeCaterina R, Sicari R, Bernini W, Lazzerini G, Buti-Strata GB, Giannessi D: Benefit/risk profile of combined antiplatelet therapy with ticlopidine and aspirin. Thromb Haemost 1991;65:504-510.

23 Executive Committee for the Asymptomatic Carotid Atherosclerosis Study: Endarterectomy for asymptomatic carotid artery stenosis. JAMA 1995;273:1421-1428.

24 Lefkovits J, Plow EF, Topol EJ: Platelet glycoprotein IIb/IIIa receptors in cardiovascular medicine. N Engl J Med 1995;332:1553-1559.

25 Mousa SA, Mu DX, Lucchesi BR: Prevention of carotid artery thrombosis by oral platelet GPIIb/IIIa antagonist in dogs. Stroke 1997;28: 830-836.

26 Guiraud-Chaumeil B, Rascol A, David J, Boneu B, Clanet M, Bierme R: Prévention des récidives des accidents vasculaires cérébraux ischémiques par les anti-aggrégants plaquettaires. Rev Neurol (Paris) 1982;138:367-385. 